\title{
Periodontal Manifestations in a Patient with Haim-Munk Syndrome
}

Kamile Erciyas ${ }^{a}$

Serhat Inaloz

A. Fuat Erciyas ${ }^{c}$

\section{ABSTRACT}

Haim-Munk syndrome is an extremely rare autosomal recessive disorder characterized clinically by palmoplantar hyperkeratosis, aggressive periodontitis with severe alveolar bone destruction, onychogryphosis, pes planus, arachnodactyly, and acro-osteolysis. Consanguinity seems a notable prerequisite. The aim of this study was therefore to report one case of this syndrome and to focus on the periodontal manifestations, in order to attract the attention of dental clinicians to this rare anomaly. (Eur J Dent 2010;4:338-340)

Key words: Periodontitis; Haim-Munk syndrome; Palmoplantar keratoderma.

\section{INTRODUCTION}

Haim-Munk syndrome (HMS) was first described among descendants of a religious isolate from India. ${ }^{1}$ Later on, some researchers considered HMS a variant of Papillon-Lefevre syndrome (PLS), on the basis of common clinical features such as abnormal hyperkeratosis of the palms and soles, and aggressive periodontitis. ${ }^{2}$

The presence of additional features including recurrent pyogenic skin infections, acroatrophic

- a Assistant Professor, Gaziantep University, Faculty of Dentistry, Department of Periodontology,

Gaziantep, Turkey.

b Professor, Gaziantep University, Faculty of Medicine, Department of Dermatology, Gaziantep, Turkey.

c Assistant Professor, Clinical of Orthodonthy, Gaziantep, Turkey.

- Corresponding author: Kamile Erciyas Gaziantep University, Faculty of Dentistry, Department of Periodontology,

Gaziantep, Turkey.

Phone: +90 3422376611

E-mail: kmlercysahotmail.com changes of nails, arachnodactyly, a peculiar radiographic deformity of the finger contapered, pointed phalangeal ends, and pes planus suggested that HMS was a distinct disorder. ${ }^{2}$ In the current study, we present a patient from a consanguineous family with severe dermatologic, periodontal, and skeletal manifestations, which are typical clinical signs of HMS.

\section{CASE REPORT}

A 15-year-old Turkish male from a consanguineous family was evaluated for aggressive periodontitis, palmoplantar hyperkeratosis, and multiple psoriasiform plaques on face, extremities, and trunk. The patient started developing thickening and scaling of the skin of the palms and soles at the age of 2-3, and shedding of primary dentition as a result of periodontitis at the age of 4 years. Secondary dentition was also affected by periodontal disease, with subsequent premature 
shedding of permanent teeth. The palmoplantar hyperkeratosis progressively worsened.

At the age of 13 years, the patient experienced arthralgia of the knees and elbows that progressively involved the rest of the large joints, including the wrists, shoulders, ankles and joints. The joints were tender and the movement was associated with pain. There was no history of swelling, redness, trauma or inflammation of the joints.

The physical examination showed severe hyperkeratosis of the palms and soles (Figure 1). The keratotic lesions of the palms extended to the lateral and dorsal aspects of the hands; besides the lesions on the soles, which were more severe, spread on to the external malleoli and on the Achilles tendon. In addition, the patient had circumscribed, scaly, and erythematous patches on the knees, elbows, and shins. He also had pes planus, and the X-ray of hands revealed typical arachnodactyly and acro-osteolysis (Figure 2).

The intraoral examination showed the presence of a few extremely mobile teeth. The gingiva around them were bright red, severely inflamed, swollen, with pus exuding from the periodontal pockets and gingival abscesses (Figure 3). The panoramic radiographs showed severe vertical and horizontal alveolar bone loss around all remaining teeth (Figure 4).

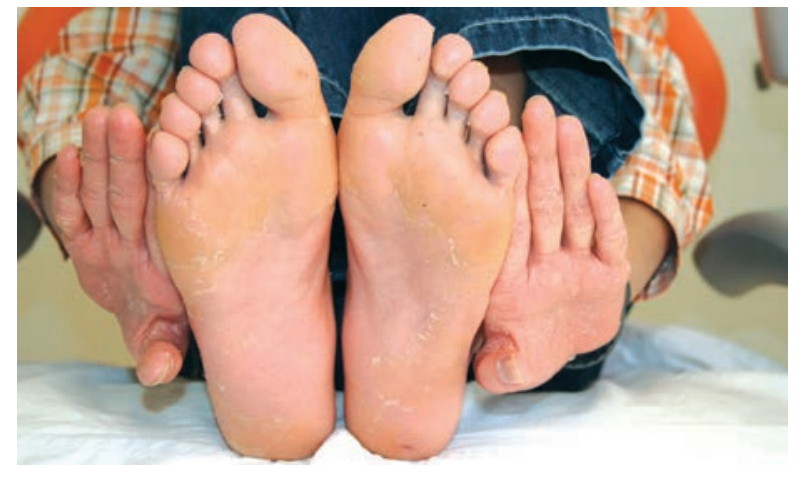

Figure 1. Palms and soles of the patient.

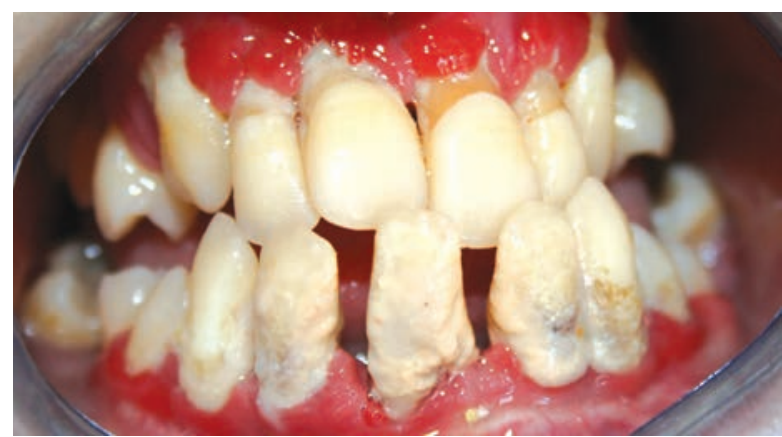

Figure 3. Intraoral view of the patient.
Blood cell count, erythrocyte sedimentation rate, liver function transaminase levels, alkaline phosphatase, total bilirubin, rheumatoid factor, antinuclear antibodies, and C3 and C4 levels were normal. Based on the patient's history, and the clinical and radiographic findings, the diagnosis of HMS was made.

The patient's family members were unaffected and no abnormal signs were present on physical examination.

Written informed consent was received from the patient.

\section{DISCUSSION}

Palmoplantar keratoderma (PPK) is a heterogeneous condition characterized by hyperkeratosis and erythema of the soles of the feet and palms of the hands. ${ }^{3}$ The PPK has been categorized into diffuse, punctate, focal, and palmoplantar ectodermal dysplasia. ${ }^{4}$ PLS and HMS are rare autoso-

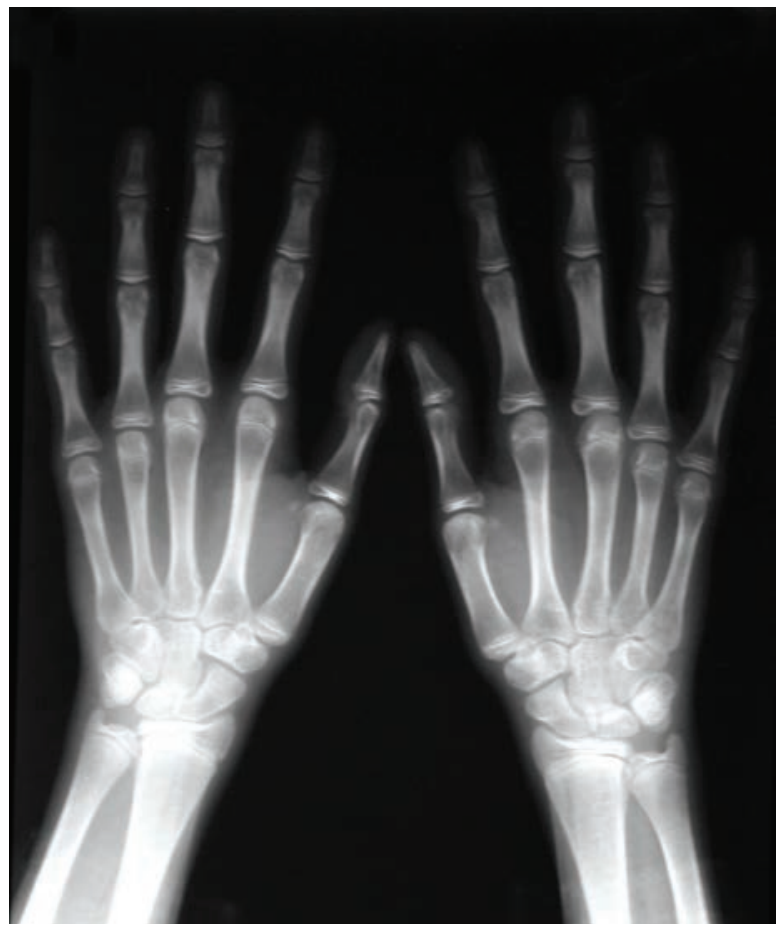
Figure 2. Hand-wrist radiographs of the patient

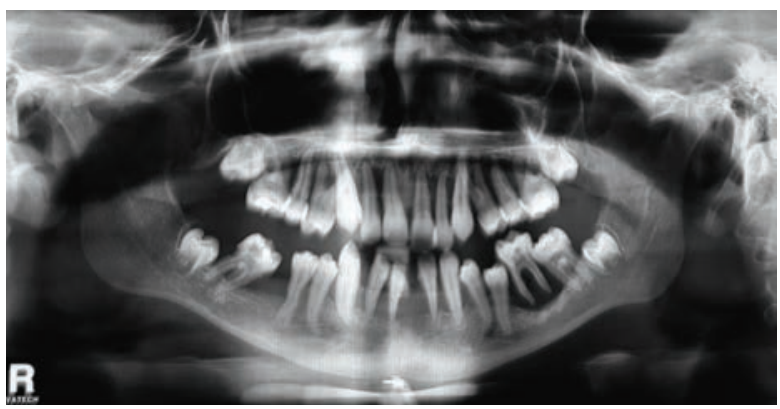

Figure 4. Panoramic radiograph of the patient. 
mal recessive type-IV PPK, and are distinguishable clinically from other types of PPK Itypes I, II, III) because of their association with aggressive periodontitis that results in tooth loss. ${ }^{2}$

Haim-Munk syndrome (HMS) was described in 4 siblings of a religious isolate from Cochin, India on the Malabar Coast, by Haim and Munk in 1965. ${ }^{1}$ In addition to palmoplantar keratosis and periodontitis, other clinical findings included recurrent pyogenic skin infections, onychogryphosis, pes planus, arachnodactyly, and acro-osteolysis. In contrast to PLS, the cutaneous findings in HMS are more severe and extensive. ${ }^{5}$ The periodontium in HMS may be less severely affected than in PLS, but gingival inflammation and alveolar-bone destruction are present and severe. ${ }^{6}$ Despite sharing common features with PLS, the existence of other clinical findings such as nail deformities and arachnodactyly suggest that HMS is a distinct disorder. ${ }^{6}$ Parenteral consanguinity is characteristic of both disorders, which are genetically related caused by allelic mutations in the lysosomal protease cathepsin C (CTSC) gene. $5,7,8$

In our case, the diagnosis of HMS was based on dermatological, periodontal, and radiological features. Moreover, the current case was also associated with consanguinity of parents.

A multidisciplinary approach is important for the care of patients with HMS and PLS. The skin manifestations are treated with topical emollients and keratolytics including salicylic acid and urea. Oral retinoids including acitretin, etretinate, and isotretinoin are the mainstay of treatment for both keratoderma and aggressive periodontitis. ${ }^{9}$ The periodontal disease in both HMS and PLS usually responds poorly to treatment. ${ }^{10}$ Effective therapy includes the extraction of the primary teeth combined with oral antibiotics and professional teeth cleaning. ${ }^{2}$

\section{CONCLUSIONS}

HMS is an extremely rare condition involving palmoplantar hyperkeratosis and aggressive periodontitis. Although these characteristic findings can be easily diagnosed, they are often overlooked. Therefore, medical and dental professionals should be aware of this rare syndrome and provide the necessary care.

\section{REFERENCES}

1. Haim S, Munk J. Keratosis palmo-plantaris congenital, with periodontitis, arachodactyly and a peculiar deformity of the terminal phalanges. Br J Dermatol 1965;77:42-54.

2. Janjua SA, Iftikhar N, Hussain I, Khachemoune A. Dermatologic, periodontal, and skeletal manifestations of Haim-Munk syndrome in two siblings. J Am Acad Dermatol 2008;58:339-344.

3. Itin $\mathrm{PH}$. Classifications of autosomal dominant palmoplantar keratoderma: past-present-future. Dermatology 1992;185:163-165.

4. Stevens HP, Kelsell DP, Bryant SP, Bishop DT, Spurr NK, Weissenbach J. Linkage of an American pedigree with palmoplantar keratoderma and malignancy Ipalmoplantar ectodermal dysplasia type III) to 17q24. Literature survey and proposed upated classification of the keratodermas. Arch Dermatol 1996;132:640-651.

5. Hart TC, Hart PS, Michalec MD, Zhang Y, Firatli E, Van Dyke TE, Stabholz A, Zlotogorski A, Shapira L, Soskolne WA. Haim-Munk syndrome and Papillon Lef'evre syndrome are allelic mutations in cathepsin C. J Med Genet 2000;37:88-94.

6. Janjua SA, Khachemoune A. Papillon Lef'evre syndrome: case report and review of the literature. Dermatol Online $J$ $2004 ; 10: 13$.

7. Hart TC, Hart PS, Bowden D, Michalec MD, Callison SA, Walker SJ, Zhang Y, Firatli E. Mutations of the cathepsin C gene are responsible for Papillon Lef'evre syndrome. $J$ Med Genet 1999;36:881-887.

8. Cury VF, Gomez RS, Costa JE, Friedman E, Boson W, De Marco L. A homozygous cathepsin C mutation associated with Haim-Munk syndrome. Br J Dermatol 2005;152:353356.

9. Siragusa M, Romano C, Batticane N, Batolo D, Schepis C. A new family with Papillon-Lefevre effectiveness of etretinate treatment. Cutis 2000;65:151-155.

10. Subramaniam P, Mathew S, Gupta KK. Papillon-Lefevre syndrome: A case report. J Indian Soc Pedodontics Dent 2008;26:171-174. 